\title{
Beryllium flux distribution and layer deposition in the ITER divertor
}

\author{
K. Schmid * \\ Max-Planck-Institut für Plasmaphysik, Boltzmannstraße 2, D-85748 Garching b. \\ München Germany
}

PACS numbers: $61.80 . J h, 52.25 . \mathrm{Vy}, 52.65,52.40 . \mathrm{Hf}$

\begin{abstract}
.
The deposition of Be eroded from the main chamber wall on the $\mathrm{W}$ surfaces in the ITER divertor could result in the formation of Be rich Be/W mixed layers with a low melting temperature compared to pure $\mathrm{W}$. To predict whether or not these layers form the Be flux distribution in the ITER divertor is required. This paper presents the results of a combination of plasma transport- with erosion/deposition simulations that allow to calculate both the Be flux distribution and the Be layer deposition in the ITER divertor. This model includes the Be source due to Be erosion in the main chamber and the deposition, re-erosion and re-deposition of Be in the ITER divertor. The calculations show that the fraction of Be in the incident particle flux in the divertor range from $\approx 10^{-3}$ to $\approx 5 \%$ with a pronounced inner outer divertor asymmetry. The flux fractions in the inner divertor are on average ten times higher than in the outer divertor. Thick Be layers only from at the inner strike point and the dome baffles. The highest Be layer growth rate is found to be $1.0 \mathrm{~nm} / \mathrm{s}$. Despite the Be deposition the formation of Be rich $\mathrm{Be} / \mathrm{W}$ mixed layers is not to be expected in ITER. The expected surface temperature at these locations during steady state operation is too low as to result in Be diffusion into $\mathrm{W}$ and thus $\mathrm{Be} / \mathrm{W}$ mixed layers can not form. The paper also discusses the influence of off normal events like ELM's or VDE's on the formation $\mathrm{Be} / \mathrm{W}$ mixed layers.
\end{abstract}

* Corresponding author (Klaus.Schmid@ipp.mpg.de) 


\section{Introduction}

Due to the large Be covered first wall area in ITER and the high physical sputtering yield of Be a large influx of Be onto the divertor surfaces can be expected. This influx of Be onto the $\mathrm{W}$ and $\mathrm{C}$ divertor tiles can lead to deposition of Be layers depending on the local Be flux fraction and Be re-erosion rate. The formation of these layers can have two negative consequences: Firstly large amounts of hydrogen can be co-deposited in these layers $[1,2,3,4,5]$ and secondly the deposition of Be on $\mathrm{W}$ surfaces can lead to the formation $\mathrm{Be} / \mathrm{W}$ alloys $[6,7]$ given that the surface temperature is high enough to allow for Be diffusion. These Be/W alloys have a melting temperature much lower than $\mathrm{W}$ which can lead to an unacceptable influx of $\mathrm{W}$ into the plasma, particularly during off normal events.

The deposition of Be eroded from a Be main wall has been investigated in [8] for the FIRE Tokamak and in [9] for the ITER Geometry. In [9] the ERO code was used to calculate the deposition of Be in the ITER divertor including re-erosion and re-deposition of Be. Due to the limitations of ERO regarding the size of the modelling volume, only the plasma volume close to the divertor strike points was included in the simulation and no global transport simulation of Be in the main chamber was performed. The flow of Be from the main chamber walls was introduced in the calculation via a constant $\mathrm{Be}$ plasma density fraction in the $\%$ range. In [8] the Be erosion from the main chamber of the FIRE Tokamak was simulated including global transport in the SOL. However the divertor fluxes were calculated taking into account neither re-erosion and re-deposition of deposited Be nor the transport of this re-eroded Be in the divertor into account. Thus so far no calculations exist for the ITER geometry that model both the main wall Be erosion, its transport into the divertor and the subsequent deposition, re-erosion and re-deposition of Be.

To predict the Be flux distribution and resulting deposition in the ITER divertor the following processes have to be modelled: The erosion of Be at the first wall of ITER, transport to the divertor and initial deposition, the re-erosion of the deposited Be and the redistribution and re-deposition of Be in the divertor. To simulate both the plasma transport- and the erosion/deposition processes two codes are coupled: The plasma transport code DIVIMP [10] is used to calculate the initial transport of Be from the main wall into the divertor and the redistribution of re-eroded Be in the divertor. The plasma surface interaction code ERODEPDIF is used to simulate the deposition of Be from plasma and the re-erosion of deposited Be. It takes into account both the kinetic processes physical sputtering and reflection and the thermally activated processes Be diffusion and sublimation.

The aim of this work is find an equlibrium flux distribution of Be and the corresponding equilibrium Be surface concentration on the ITER divertor surfaces. Given these distributions and the temperatures in the ITER divertor one can determine where potentially Be rich Be/W alloys may form. Also based on literature data for the Behydrogen co-deposition $[1,2,3,4,5]$ one can estimate the potential $\mathrm{T}$ co-deposition in the ITER divertor.

To simplify the erosion/deposition calculations an ITER with a full W divertor is 
considered in the calculations. This option is currently discussed to rid ITER from the issue of carbon-hydrogen co-deposition. The absence of carbon excludes chemical erosion and diffusion in ternary alloys from the model. Since with a full $\mathrm{W}$ divertor no $\mathrm{C}$ source exists in the machine, a plasma solution without $\mathrm{C}$ but $\mathrm{Ar}$ as main radiating impurity is chosen. Except for the main radiating impurity this plasma background, taken from a B2/EIRENE calculation [11], corresponds to high power (130MW power into the SOL) ITER reference discharge. Only a little number of plasma backgrounds for ITER without $\mathrm{C}$ as main radiating impurity are available. In particular no Deuterium/Tritium background plasma with $\mathrm{Ar}$ as as main radiating impurity is available. Therefore this work is limited to a D plasma background. The details of the plasma background used here are given in [11]

\section{Simulation details}

The primary goal of these modelling calculations is to find a poloidally resolved, equilibrium Be flux distribution in the ITER divertor together with the corresponding equilibrium Be layer growth or $\mathrm{W}$ erosion rate. To find these equilibrium values the plasma transport code DIVIMP and the plasma surface interaction code ERODEPDIF are coupled. The details of DIVIMP plasma transport and the ERODEPDIF erosion/deposition/diffusion/sublimation calculations are described in detail in 2.2 and 2.1 respectively.

For the coupled calculations the ITER divertor was subdivided into 15 elements as shown in Fig. 1. The numbers at each element in Fig. 1 is the DIVIMP wall element index. The numbers are not continuous since for modelling the surface processes the divertor geometry was more coarsely discretized than internally used by DIVIMP. The equilibrium poloidal flux distribution $\Gamma^{E Q, i}$ on these elements is given by the sum of the incident flux of Be from the main chamber $\Gamma_{\text {Main }}^{i}$ and the flux of Be due to redistribution $\Gamma_{R e d}^{E Q, i}$ of Be re-eroded from Be deposited in the divertor. The index i thereby refers to the i-th of the 15 elements. $\Gamma_{\text {Main }}^{i}$ is constant and does not depend on the Be deposition on the $\mathrm{W}$ divertor elements. It only depends on the physical erosion of the Be main wall by the plasma flux and subsequent SOL transport, both determined by the chosen B2/EIRENE plasma background. In contrast $\Gamma_{R e d}^{E Q, i}$ depends on the Be reerosion flux $\Phi^{E Q, i}$ from deposited Be layers and how this Be is redistributed through plasma transport. $\Phi^{E Q, i}$ depends on the equilibrium Be surface composition $C_{B e}^{E Q, i}$ on the i-th element. $C_{B e}^{E Q, i}$ in turn depends on the deposition of Be due to $\Gamma^{E Q, i}$ and erosion of Be due to the constant, incident total background plasma flux $\Gamma_{B k n d}^{i}$. To find these equilibrium values an iterative scheme was applied:

In the first iteration a pure $\mathrm{W}$ divertor was assumed (i.e $C_{B e}^{i} \equiv 0$ ) and thus the current Be flux on the i-th divertor tile $\Gamma^{i}$ is given by $\Gamma_{\text {Main }}^{i}$ alone, since no Be re-erosion takes place in the divertor. Based on this $\Gamma^{i}$ and $\Gamma_{B k n d}^{i}$ ERODEPDIF is used to calculate the current equilibrium surface composition $C_{B e}^{i}$ on each of the 15 tiles (see 2.1). Based on $C_{B e}^{i}$ and $\Gamma_{B k n d}^{i}$ the re-erosion flux $\Phi^{i}$ from the i-th element is calculated according to eq. 1.

$$
\Phi^{i} \quad=\Gamma_{B k n d}^{i} Y_{B e}^{i}\left(C_{B e}^{i}\right) C_{B e}^{i}\left(m^{-2} s^{-2}\right)
$$


$\mathrm{Y}_{B e}^{i}\left(C_{B e}^{i}\right)=$ Total Be sputter yield at tile i. See also 2.1

From $\Phi^{i}$ the re-deposition $\Gamma_{\text {Red }}^{i}$ on each of the 16 elements is calculated according to eq. 2 .

$$
\begin{aligned}
\Gamma_{\text {Red }}^{i}= & \sum_{j=1}^{16} \xi_{i j} \Phi^{j}\left(m^{-2} s^{-2}\right) \\
\xi_{i j}= & \text { Flux redistribution matrix. See also } 2.2 \\
& \text { Fraction of flux from element } \mathrm{i} \text { that hits element } \mathrm{j}
\end{aligned}
$$

From $\Gamma_{\text {Red }}^{i}$ the new $\Gamma^{i}$ after the current iteration step is calculated according to 3

$$
\begin{aligned}
& \tilde{\Gamma}^{i}=\Gamma_{\text {Main }}^{i}+\Gamma_{\text {Red }}^{i}\left(m^{-2} s^{-2}\right) \\
& \tilde{\Gamma}^{i}=\text { New Be flux on element i after the current iteration }
\end{aligned}
$$

After each iteration $\tilde{\Gamma}^{i}$ is compared to $\Gamma^{i}$ and if both are equal the iteration stops and the last values for $\Gamma^{i}$ and $C_{B e}^{i}$ are then assumed to be the equilibrium values $\Gamma^{E Q, i}$ and $C_{B e}^{E Q, i}$. If they are not equal $\Gamma^{i}$ is replaced by $\tilde{\Gamma}^{i}$ and the next iteration is performed. While this approach finds equilibrium values it does not describe the full time evolution of the Be surface composition or the Be flux distribution. At each iteration both DIVIMP and ERODEPDIF calculate equilibrium values given the current state of the system: ERODEPDIF calculates equilibrium $C_{B e}^{i}$ and $\Phi^{i}$ values for the current $\Gamma^{i}$. DIVIMP calculates the fraction of flux from element $i$ that is deposited on element $j$ in equilibrium corresponding to the current $\Phi^{i}$. This redistribution is described in eq. 2 through $\xi_{i j}$ which is determined from DIVIMP as explained in section 2.2. Since the Be plasma concentration are small enough not to influence the background plasma neither $\xi_{i j}$ nor $\mathrm{Y}_{B e}^{i}\left(C_{B e}\right)$ depend on the current state of the system and are constant for each iteration. In the calculation the $\xi_{i j}$ matrix is different for each Be charge state ranging from neutral $\mathrm{Be}$ to $\mathrm{Be}^{+4}$. This allows to resolve the redeposited spectrum into different charge states which have different energies after the sheath acceleration and thus result in different sputtering yields. This detail has been omitted in the above equations for simplicity.

\subsection{Be layer deposition}

To simulate the deposition of $\mathrm{Be}$ on a $\mathrm{W}$ divertor both the kinematic processes: physical sputtering and reflection and the thermally activated processes: diffusion and sublimation have to modelled. These two types of processes occur on very different time scales making a simultaneous description difficult. In previous approaches [12] kinetic monte carlo codes like TRIDYN [13] were coupled to diffusion codes in an iterative way. These coupled approaches require numerous $(\approx 1000)$ individual TRIDYN calculations. This would have been impossible here because due to the small Be concentrations in the incident particle flux a large number $\approx 10^{7}$ of particles has to be traced in each TRIDYN run to achieve proper statistics, resulting in unacceptable calculation times. To circumvent the calculation time problem the ERODEPDIF code was developed. It models the kinetic processes not through monte carlo simulation but through predetermined total sputter $Y$ and reflection yiels $R$. Diffusion is modelled using Fick's 
second law and sublimation based on sublimation fluxes calculated from literature vapour pressure data [14]. For the bombardment of a W surface with Be the total sputter yields and reflection yields depend on the composition in a Be / W mixed surface. This is due to the dominant physical sputtering process in this system: The incident particle e.g $\mathrm{D}$ penetrates into the surface and is reflected at a depth of $\approx 1$ to $3 \mathrm{~nm}$ for the typical particle energies encountered in the ITER divertor. On its way out of the surface the particle sputters atoms in a single forward collision. This sputtering process is much more efficient than collisions with atoms on the way into the surface. The recoils generated on the way into the surface have to undergo at least one additional collision to invert their impulse and leave the surface. Since the dominant sputtering process involves the reflection of the incident projectile it is very sensitive to the concentration of $\mathrm{Be}$ and $\mathrm{W}$ in the subsurface region, since small amounts of $\mathrm{W}$ greatly enhance the reflection probability.

ERODEPDIF simulates the exposure of a divertor element surface to a flux $\Gamma^{i}\left(m^{-2} s^{-1}\right)$ for a time $t_{\text {Total }}(s)$ up to total fluence $\Theta\left(\mathrm{m}^{-2}\right)$ in small discreet fluence(time) steps $\mathrm{d} \Theta$ $(\mathrm{dt})$. The fluence steps $\mathrm{d} \Theta$ are calculated from $\mathrm{dt}$ based on the incident flux $\Gamma^{i}$ from eq. 3. The surface is subdivided into three consecutive depth zones: The sputter zone (SZ) the reaction zone (RZ) and the diffusion zone (DIFFZ).

The application of each step $\mathrm{d} \Theta(\mathrm{dt})$ is performed in two consecutive steps: In the first step the erosion of Be and $\mathrm{W}$ and the deposition of Be in the sample surface is calculated. In the second step the diffusion of the Be depth profile is calculated.

Erosion due to sputtering and sublimation only occurs in the SZ and deposition only in the RZ. Both the SZ and RZ consist of a single layer each. The net change in areal density of Be and $\mathrm{W}$ in the $\mathrm{SZ} \Delta \delta_{B e, W}^{S Z}$ is calculated according to eq. 4, 5 and the net change of the Be areal density in the $\mathrm{RZ} \Delta \delta_{B e}^{R Z}$ is calculated according to 6 . Diffusion of the Be depth profile occurs throughout the target including the layers in all three zones.

$$
\begin{aligned}
\Delta \delta_{B e}^{S Z}= & -\Gamma_{B k n d}^{i} d t Y_{B e}^{i} C_{B e}^{i}-\psi_{B e}\left(T, C_{B e}^{i}\right) d t+ \\
& \Gamma^{i} d t\left(1-R^{i}\right) \\
= & -\Gamma_{B k n d}^{i} d t Y_{W}^{i} C_{W}^{i}-\psi_{W}\left(T, C_{W}^{i}\right) d t \\
\Delta \delta_{W}^{S Z}= & \Gamma^{i} d t\left(1-R_{B e}^{i}\right) \\
\Delta \delta_{B e}^{R Z}= & \text { Surface concentration of Be or W respectively } \\
C_{B e, W}^{i} & \text { Total sputter yield of Be or W respectively } \\
Y_{B e, W}^{i} & \text { for the current surface composition } \\
= & \text { Reflection yield of Be } \\
& \text { for the current surface composition } \\
R_{B e}^{i} & \text { Sublimation flux of Be or W respectively } \\
& \text { at temperature T }
\end{aligned}
$$

As explained above the total sputter yield $Y_{B e, W}^{i}$ depends on the subsurface concentration of $\mathrm{Be}$ in the $\mathrm{RZ}$ via the reflection probability and on the surface concentration of Be in the SZ, since it requires near surface Be atoms to be kicked out by the reflected projectile. In addition the total sputter yield still depends on the 
energy as describe by the Bohdansky formula [15]. As an example of the concentration dependence Fig. 2 shows the total sputtering yield of $\mathrm{Be}$ in $\mathrm{Be} / \mathrm{W}$ mixed surface due to $40 \mathrm{eV}$ D ions as function of the reaction $\left(C_{B e}^{R Z}\right)$ and sputter zone $\left(C_{B e}^{S Z}\right)$ Be concentrations. One can see that for low Be concentrations in both the reaction and sputter zone the Be sputter yield is higher by almost an order of magnitude compared to the value of pure Be. The data in Fig. 2 shows that the sputter yield is influenced by the fraction of Be in the RZ due to the change in reflection of the eroding species. Lower values meaning higher $\mathrm{W}$ content and thus higher reflection leading to stronger Be sputtering. A similar dependence also holds for the $\mathrm{W}$ sputter yield and for the Be reflection yield $R_{B e}$ from a Be/W mixed surface. To model this complex dependence of the sputter and reflection yields on the surface composition a parameter scan was performed using TRIDYN: The particle energy E, $C_{B e}^{S Z}$ and $C_{B e}^{R Z}$ were varied throughout the entire possible value space for each of the incident species, D, He, Ar and Be. In these TRIDYN calculations the SZ was defined as a $1 \mathrm{~nm}$ wide layer at the surface and the RZ as a $3 \mathrm{~nm}$ wide layer just below the sputter zone. The resulting tables for each of the incident species species of $\tilde{Y}_{B e, W}^{D, H e, A r, B e}\left(E, C_{B e}^{S Z}, C_{B e}^{R Z}\right)$ are then trilinearly interpolated to obtain values for a given set of $\left(E, C_{B e}^{S Z}, C_{B e}^{R Z}\right)$. The total sputter yield for Be respectively $\mathrm{W}$ is then calculated according to equation 7 .

$$
\begin{aligned}
& Y_{B e, W}^{i}=\sum_{\text {Element }}^{D, H e, A r, B e} \sum_{q=1}^{q_{M a x}} \zeta_{E l e m e n t}^{q} \tilde{Y}_{B e, W}^{\text {Element }}\left(E, C_{B e}^{S Z}, C_{B e}^{R Z}\right) \\
&= \text { Fraction of Element at charge state q in incident flux } \\
& \zeta_{\text {Element }}^{q \text { lement }}\left(\mathrm{E}, C_{B e}^{S Z}, C_{B e}^{R Z}\right)= \text { Total sputter yield of Be or W for Element and } \\
& \tilde{Y}_{B e, W}^{\text {Element }}\left(\mathrm{Surface} \text { state } C_{B e}^{S Z}, C_{B e}^{R Z}\right. \text { and energy E }
\end{aligned}
$$

The sublimation flux of $\mathrm{W}$ or Be depends both on the surface temperature and on the composition of the surface since the surface binding energies of Be or $\mathrm{W}$ varies with composition as shown in eq. 9.

$$
\begin{aligned}
\psi_{B e, W}\left(T, C_{B e}^{i}\right)= & \psi_{B e, W}^{0} \exp \left(\frac{-E_{S u b l}^{B e, W}\left(C_{B e}^{i}\right)}{K_{B} T}\right) \\
\psi_{B e, W}^{0} & \text { Constant flux factor for Be and W respectively }\left(m^{-2} s^{-1}\right) \\
E_{S u b l}^{B e, W}\left(C_{B e}^{i}\right)= & \text { Composition dependent sublimation energy } \\
& \text { for Be and W respectively }(\mathrm{eV})
\end{aligned}
$$

No literature data on the dependence of the sublimation energies of Be $E_{S u b l}^{B e}\left(C_{B e}^{i}\right)$ or $\mathrm{W} E_{S u b l}^{W}\left(C_{B e}^{i}\right)$ in Be/W alloys exist to our knowledge. Therefore the sublimation energy of $\mathrm{Be}$ and $\mathrm{W}$ in a $\mathrm{Be} / \mathrm{W}$ mixture was calculated according to eq. 10 and 11 following an approach [16] commonly used in TRIDYN sputtering calculations to determine surface binding energies.

$$
\begin{aligned}
& E_{\text {Subl }}^{B e}\left(C_{B e}^{i}\right)=E_{\text {Subl }}^{B e, B e} C_{B e}^{i}+\left(1-C_{B e}^{i}\right) E_{\text {Subl }}^{B e, W} \\
& E_{\text {Subl }}^{W}\left(C_{B e}^{i}\right)=E_{\text {Subl }}^{W, W}\left(1-C_{B e}^{i}\right)+C_{B e}^{i} E_{\text {Subl }}^{W, B e}
\end{aligned}
$$




$$
E_{S u b l}^{i, j} \quad=\text { Binding energy of element } \mathrm{i} \text { on an element } \mathrm{j} \text { surface }
$$

Following the idea in $[16,17]$ the $E_{\text {Subl }}^{W, B e}$ and $E_{\text {Subl }}^{B e, W}$ heterogenous surface binding energies were calculated from the sublimation energies of the pure materials according to eq. 12 .

$$
E_{\text {Subl }}^{i, j}=\frac{E_{\text {Subl }}^{i, i}+E_{\text {Subl }}^{j, j}}{2}
$$

The pure element sublimation energies were taken from [14]: $E_{\text {Subl }}^{B e, B e}=3.2 e V, E_{\text {Subl }}^{W, W}=$ $8.68 \mathrm{eV}$.

As the areal densities of Be and W change in the SZ and RZ the layer thicknesses would have to change. Since the SZ and RZ layer thicknesses were chosen such that erosion and deposition occur on realistic length scales for the energies of the incident particles, their thicknesses have to be kept constant during the calculation: If net deposition occurs in the RZ material is moved from the RZ into the DIFFZ. The DIFFZ consists of a number of layers sampling the Be depth profile. If material is moved into the DIFFZ it is stored in the first layer of the DIFFZ. If there is net erosion in the SZ material is moved from the RZ into the SZ and the material loss in the RZ is restored from the first layer in the DIFFZ. If during this material transfer the thickness of the first layer in the DIFFZ increases above twice its initial width, it is split into two layers. If its thickness drops below half its initial width the layer is merged with the second layer in the DIFFZ. With this mechanism of splitting and merging the growth of deposited layers or the net erosion of the divertor element surface is simulated. It is identical to the method used in TRIDYN to calculate layer growth and surface recession.

The diffusion of the Be depth profile is simulated by solving the diffusion equation 13 .

$$
\begin{aligned}
\frac{\partial C_{B e}^{i}(x, t)}{\partial t}= & \frac{\partial C_{B e}^{i}(x, t)}{\partial x} * \frac{\partial D(x, t, T)}{\partial x}+D(x, t) * \frac{\partial^{2} C_{B e}^{i}(x, t)}{\partial x} \\
D(x, t, T)= & \text { The concentration and temperature dependent } \\
& \text { interdiffusion coefficient of Be and } \mathrm{W}
\end{aligned}
$$

A finite difference scheme described in [18] is applied to solve eq. 13 to obtain the diffusion profile after the current time step dt. The diffusion data for Be and $\mathrm{W}$ is sparse. To obtain a reasonable estimate for the concentration and temperature dependent diffusion coefficient $D\left(C_{B e}(x, t), T\right)$ it was assumed that $D\left(C_{B e}(x, t), T\right)$ could be written as in eq. 14 .

$$
\begin{array}{ll}
D\left(C_{B e}(x, t), T\right) & =\frac{D_{0}\left(C_{B e}(x, t)\right)}{\alpha} \exp \left(\frac{-E_{D}}{K_{B} T}\right) \\
D_{0}\left(C_{B e}(x, t)\right) & =\text { Concentration dependence }\left(m^{2} s^{-1}\right) \\
E_{D} & =\text { Activation energy for diffusion }(\mathrm{eV}) \\
\alpha & =\text { Scale factor }
\end{array}
$$

To determine $D_{0}\left(C_{B e}(x, t)\right)$ equation 13 was used to model the $\mathrm{Be} / \mathrm{W}$ interdiffusion profiles from Fig. 1 in [6]. These interdiffusion profiles were obtained by heating a $\mathrm{Be} / \mathrm{W}$ diffusion couple at $1070 \mathrm{~K}$ for different times. Similar to an approach in [12] a Gauss function was used as the principal shape of $D_{0}\left(C_{B e}(x, t)\right)$. In the calculation three 
parameters (center, full width and height) of the Gauss function were varied until these depth profiles could be reproduced from an initial Be step profile. The best values were found to be: center $=0.9$, full width $=0.05$, height $=5 \times 10^{-20}\left(\mathrm{~m}^{2} \mathrm{~s}^{-1}\right)$. To estimate $E_{D}$ the diffusion coefficient estimates from [19] Fig. 2 where fitted to an arrhenius function yielding an activation energy of $4.5 \mathrm{eV}$. The $\alpha$ parameter was used to match the temperature dependence data with the concentration dependence data obtained for 1070K. $\alpha$ was chosen such that $D\left(C_{B e}(x, t), 1070 K\right)=D_{0}\left(C_{B e}(x, t)\right)$.

To validate ERODEPDIF a comparison with TRIDYN was performed. Both programs were used to simulate the deposition of $\mathrm{Be}$ on $\mathrm{W}$ at low temperatures where diffusion is negligible and Be layer deposition is dominated by sputtering and reflection. The incident particle spectrum was chosen such that it would mimic the average conditions at the divertor entrance baffles as obtained from the B2/E background plasma: $0.3 \% \mathrm{Ar}$ at $390 \mathrm{eV}, 1 \%$ He at $125 \mathrm{eV}, 93.7 \% \mathrm{D}$ at $90 \mathrm{eV}$ and $5 \%$ Be at $140 \mathrm{eV}$. The Be fraction of $5 \%$ is higher than the average Be fraction due to the main wall source alone («1\%) in order not to run into calculation time problems in the TRIDYN calculations. The exposure of an initially pure $\mathrm{W}$ surface to a total fluence $(\mathrm{D}+\mathrm{Be}+\mathrm{He}+\mathrm{Ar})$ of $1 \times 10^{23}\left(\mathrm{~m}^{-2}\right)$ was simulated. In the TRIDYN calculation $2 \times 10^{6}$ particles were traced to sample the incident particle spectra correctly. In Fig 3 the Be depth profiles calculated by ERODEPDIF and TRIDYN for different fluences are compared. The depth profiles are in good agreement to predict Be layer formation.

\subsection{Be main wall erosion and plasma transport}

The DIVIMP plasma transport calculation operates in the "trace limit" approximation: The concentrations of the traced impurities, Be in this case, are low enough such that they do not influence the background plasma parameters. In this work DIVIMP is used simulate two transport cases: The transport of the eroded Be from the main chamber SOL down into the divertor and the redistribution of Be that is eroded from deposited layers in the divertor.

To calculate the transport of Be from the main chamber into the divertor, first the $\mathrm{Be}$ erosion fluxes at the main wall have to be calculated. DIVIMP was initially designed to simulate the erosion and transport of $\mathrm{C}$ in divertor and SOL regions of tokamaks. Therefore, it can only treat erosion at the divertor targets and is limited to one incident eroding and one target/impurity species. The main wall is not part of DIVIMP's calculation grid and thus the erosion of Be from these surfaces cannot be handled directly by DIVIMP. Also the erosion by multiple plasma species (D-CX, $\mathrm{D}^{+}, \mathrm{He}^{+X}$, $\mathrm{Ar}^{+X}$ ) cannot be handled by DIVIMP. Therefore the Be erosion calculation had to be performed in a separate step. The particle fluxes of D-CX, $\mathrm{D}^{+}, \mathrm{He}^{+X}, \mathrm{Ar}^{+X}$ across the grid boundary, and the plasma parameters Te, Ti at the grid boundary are known from the B2/Eirene calculations and can be extrapolated towards the wall. While the CX flux can be readily extrapolated towards the wall without having to account for attenuation processes, the extrapolation of the ion fluxes and plasma parameters Te, Ti to the wall is not straight forward. Since no validated physical model exists on how to extrapolate towards the wall, we assumed no attenuation at all during these calculations. Therefore 
the calculated Be erosion fluxes are an upper boundary. The DIVIMP calculation grid and the main wall geometry are shown in Fig. 4. The maximum distance between the DIVIMP calculation grid and the first wall is shown in 5 . It varies from 0 at the strike points to a maximum of $\approx 0.3 \mathrm{~m}$ at the lower end of the inner heat shield. This gap influences the transport calculation of eroded Be from the main chamber to the divertor. Be that leaves the calculation grid somewhere in the main chamber is assumed to be deposited at the closest position on the first wall. In reality it would travel along field lines that end somewhere on the lower part of the main wall or at the divertor entrance baffles. Therefore in reality the Be flux onto the divertor entrance baffles will be higher than in predicted by the DIVIMP simulation. The fraction ending up deeper down in the divertor should be modelled correctly since the field lines ending there are part of the DIVIMP calculation grid.

Based on the CX and ion fluxes on the wall elements, the Be-erosion flux was calculated using sputter yields from the revised Bohdansky formula [15]. The energy of an ion with charge state $\mathrm{q}$ was calculated using $E_{I O N}=3 q T_{e}+2 T_{i}$ and the CX energy was taken directly from the B2/Eirene background plasma solution. Based on the so calculated distribution of the Be erosion flux $\left(m^{-2} s^{-1}\right)$ along the first wall, impurity launch probabilities along the first wall were calculated as input into DIVIMP. In DIVIMP Be atoms are then launched with a poloidal distribution according to these input probabilities. The particles are then followed through the subsequent impurity transport processes. Each of the Be atoms is followed until it hits the first wall or divertor. This impact location along with the Be charge state is recorded yielding a poloidal and charge state resolved impact distribution. Since each launched Be atom corresponds to a fraction of the total Be influx into the plasma due the calculated main wall erosion the impact distribution can be converted into $\Gamma_{\text {Main }}^{i}$.

To determine the Be redistribution matrix $\xi_{i j}$ separate DIVIMP calculations were performed for each of the 15 divertor elements in Fig. 1. As already indicated above the $\xi_{i j}$ matrix is charge resolved with $\xi_{i j}^{q}$ being the fraction of Be emitted from element $\mathrm{i}$ that hits element $\mathrm{j}$ in charge state $\mathrm{q}$. To determine a single matrix element $\xi_{i j}^{q}$ a fixed flux of neutral Be was emitted into the divertor plasma homogeneously along element i. It was then monitored which fraction of this emitted flux ends up on element $\mathrm{j}$ at charge state q. This fraction then corresponds to the matrix element $\xi_{i j}^{q}$. Since the Be fractions are low enough not to disturb the background plasma the $\xi_{i j}^{q}$ matrix is constant and does not change with the current state of the system described by $C_{B e}^{i}$ and $\Gamma^{i}$. The flux distribution matrix is shown in Fig. 6. For simplicity the sum over all charge states is shown as gray scale values in Fig. 6. The x-axis shows the indices (see Fig. 1) of the source divertor elements and the y-axis the indices (see Fig. 1) of the destination elements. The diagonal dotted line indicates the $\xi_{i j}$ values that correspond to local re-deposition which is the dominant re-deposition processes. The inner divertor element indicated by the solid line collects Be from all the other divertor elements, whereas the outer divertor element indicated by the dashed line only collects Be from the outer divertor baffle area. Summing over all $\xi_{i j}$ matrix elements gives the divertor retention for Be, which is $\approx 95 \%$ 


\section{Results and discussion}

\subsection{Be main wall erosion}

As part of the main wall Be erosion calculation the ion fluxes and plasma temperatures were extrapolated from the boundary of the DIVIMP calculation grid towards the ITER first wall. As an example Fig. 7 a.) shows the resulting D ion energies and Fig. 7 b.) the $\mathrm{D}$ ion Fluxes at the first wall. At at the Be main chamber wall the D ion fluxes are in the range from $10^{19}$ to $10^{20}\left(\mathrm{~m}^{-2} \mathrm{~s}^{-1}\right)$ and the $\mathrm{D}$ ion energies are of the order of a few hundred $\mathrm{eV}$. These flux and ion energy values at the first wall match those published in [20]. Be Erosion at the first wall is dominated by D and Ar ions. In Fig. 8 the average Ar ion flux and average Ar ion energy are shown. The poloidal distribution of the Ar ion flux is similar to that of the $\mathrm{D}$ ions but is smaller by 3 to 4 orders of magnitude. In Fig. 9 the D-CX flux and average D-CX energy are shown. The poloidal distribution of D-CX flux is similar to that of the D ions. The highest flues occur in the divertor with energies below $10 \mathrm{eV}$. The highest average D-CX energies are found in the main chamber but the CX-flux is much lower.

The Be main wall erosion fluxes calculated from the extrapolated wall fluxes and plasma temperatures are shown in Fig. 10. The average Be erosion flux is $6 \times 10^{18}\left(\mathrm{~m}^{-2} \mathrm{~s}^{-1}\right)$ which corresponds to $\approx 0.05 \mathrm{~nm} / \mathrm{s}$. It shows a pronounced minimum at the location of the upper divertor due to the low plasma temperatures at this location. The total amount of eroded Be at the main wall when integrated over the entire surface area of the first wall amounts to $2 x 10^{21} \mathrm{Be} / \mathrm{s}$.

\subsection{Be influx and deposition in the divertor}

The poloidally integrated total erosion of Be in the main chamber results in an influx of $5.4 \times 10^{19} m-t^{-1} r^{-1}$ into the main chamber plasma. The unit $m-t^{-1} s^{-1}$ thereby stands for Be particles per toroidal meter and second owing to the integration along the ploidal direction. The initial Be flux into the divertor is $1.1 \times 10^{19} \mathrm{~m}-\mathrm{tor}^{-1} \mathrm{~s}^{-1}$ or $\approx 20 \%$ of the Be eroded in the main chamber. The rest of the main chamber Be source is re-deposited in the main chamber.

The fraction Be in the particle flux on the divertor $\Gamma_{M a i n}^{i} / \Gamma_{B k n d}^{i}$ calculated with DIVIMP due to main chamber Be erosion as the only source is shown in Fig. 11: The square symbols show the charge resolved Be flux distribution and the open circle to total Be flux due to main wall erosion alone without the influence of re-deposition. The open circles show a strong inner outer divertor asymmetry. The flux fractions at the inner divertor are higher than in the outer divertor. The maximum flux fraction of 0.06 is found at the baffle at the inner divertor entrance. The maximum Be flux fraction in the outer divertor is roughly an order of magnitude lower. No Be flux at all is found on the outer part of the dome baffle. The Be flux fraction in most of the divertor is well below the value of $10^{-3}$ that was assumed in [9]. The reason for the inner-outer divertor asymmetry is due preferential transport of Be to inner divertor due to the friction force exerted by the background plasma flow. In the background plasma solution in use no artificial flows were introduced only the Pfirsch-Schlüter flows were present. 
Based on this Be flux distribution ERODEPDIF was used to calculate the Be layer deposition in the divertor, without the influence of re-erosion and re-deposition. The calculations also did not involve diffusion or sublimation since for steady state conditions the surface temperatures are too low as to result in significant sublimation or diffusion. In Fig. 12 the equilibrium erosion/deposition rates resulting from this calculation are shown as open symbols. Most of the divertor is erosion dominated with erosion rates ranging up to $0.1 \mathrm{~nm} / \mathrm{s}$ at the outer divertor strike point. Net deposition only occurs at inner divertor baffle where the Be flux fractions are highest (see Fig. 11) and at the inner part of the dome baffle where the particle energy is so low (see Fig. 7 a.)) that no Be erosion occurs.

The situation changes when one also takes into account the re-erosion and re-deposition processes in the divertor as explained in section 2. Again diffusion and sublimation were not taken into account in the ERODEPDIF part of the iteration. In contrast to the case where the main wall was the only Be source, now the re-erosion of Be produces new Be sources inside the divertor. For instance the strong Be re-erosion from the outer divertor strike point area, leads to an influx of Be also onto the dome baffle as depicted in Fig. 11 as filled circles. The Be flux fractions on the rest of the divertor area also increase by up to two orders of magnitude. Generally the Be flux fraction distribution becomes more homogenous when re-erosion and re-deposition processes are included. These variations in the Be flux distribution result in a drastic change in the equilibrium erosion / deposition rates on the dome baffle shown as filled symbols in Fig. 12. Due to the low particle energies there, Be is not re-eroded and thus continuous layer growth occurs with a closed Be surface with a peak growth rate of $\approx 1 \mathrm{~nm} / \mathrm{s}$. Also at inner divertor entrance baffle the increase of the Be influx in combination with the low particle energies leads to a higher deposition rate. In the outer divertor despite the increase in the Be influx no closed Be surface layer forms due to the strong re-erosion of Be and thus the outer divertor remains a net erosion zone.

In summary taking into account re-erosion and re-deposition leads to fully Be covered surface areas at the inner divertor entrance baffle point and at the dome baffle. The highest net growth rate of Be layers of $1 \mathrm{~nm} / \mathrm{s}$ is found on the dome baffle. The surface temperature at these locations during steady state operation is well below $900 \mathrm{~K}$. According to [6] the formation of Be/W alloys a surface temperature of around $900 \mathrm{~K}$ is required for diffusion to become high enough to form $\mathrm{Be} / \mathrm{W}$ alloys. At lower temperatures the formation of these alloys is kinetically hindered. Thus in steady state conditions no Be/W alloy formation is expected due to the low surface temperature. At the outer divertor where the temperature would be sufficient no Be is deposited due to the strong Be erosion.

\subsection{Influence of temperature excursions}

While no Be/W alloy formation is expected for steady state conditions due the low expected surface temperatures, ELM's or VDE's will lead to temperatures high enough for diffusion and $\mathrm{Be} / \mathrm{W}$ phase formation. However these temperature excursions also lead to strong sublimation of Be and therefore the question arises which process will 
dominate: diffusion and phase formation or sublimation. As explained in section 2.1 the input data for simulating diffusion and sublimation in the Be/W system is sparse. The values used in the simulations shown here represent the best estimate given the available data. For precise predictions additional experiments are necessary. Never the less they allow to estimate whether Be rich Be/W alloys will form during temperature excursions or sublimation will lead to evaporation of the deposited Be layers.

The location where the alloy formation is most likely to occur is the inner divertor entrance baffle where the Be influx is high and re-erosion is moderate. Therefore temperature excursions at the inner divertor entrance baffle were chosen as a reference scenario. ELM's and VDE's can lead to a range of temperatures. However with respect to alloy formation only the range from $900 \mathrm{~K}$ to $1500 \mathrm{~K}$ is of interest since below $900 \mathrm{~K}$ no alloy formation takes place and above $1500 \mathrm{~K}$ Be melts leading to loss of material and thus also hindering Be/W alloy formation. Therefore to investigate if $\mathrm{Be} / \mathrm{W}$ alloys form within this temperature window a calculation was performed at $1500 \mathrm{~K}$ to maximize the alloy formation rate but also maximize the evaporative loss of Be. If they form at $1500 \mathrm{~K}$ the same should also occur at lower temperatures where the evaporative losses are even smaller. Therefore an ERODEPDIF calculation was performed for divertor element number 142 (see Fig. 1) using the determined equilibrium Be plasma flux fraction $\Gamma^{E Q, 142} / \Gamma_{B k n d}^{142} \approx 3 \%$ and the incident particle spectrum appropriate for this divertor location. During the ERODEPDIF calculation the surface temperature was initially kept at $600 \mathrm{~K}$ and a $20 \mathrm{~nm}$ Be layer was grown. Then in a subsequent calculations the surface temperature was increased linearly during $10^{-5} \mathrm{~s}$ to $1500 \mathrm{~K}$ respectively. It then decayed back exponentially to its initial value of $600 \mathrm{~K}$ during $8 \times 10^{-4} \mathrm{~s}$. This time evolution of temperature corresponds approximately to that of an ELM at the inner target in JET taken from Fig. 6 in [21]. The base temperature was taken from Fig. 5 in [22]. The maximum temperature was limited to $1500 \mathrm{~K}$ since above this temperature $\mathrm{Be}$ and some of the $\mathrm{Be} / \mathrm{W}$ alloys become liquid [23]. No spatial gradients of the temperature were taken into account, the whole depth profile was assumed to be at the same temperature. The rest of the conditions remained constant which is an approximation since during ELM's or VDE's also the particle spectrum (composition and energy) will differ from that during steady state conditions. In Fig. 13 the Be depth profiles after the growth phase and after the temperature excursions are shown. For the temperature excursion calculations two different sublimation energy models were used: One was calculated using the constant sublimation energy of pure Be $\left(E_{\text {Subl }}^{B e}=\right.$ const. $\left.=3.2 \mathrm{eV}\right)$ depicted as open symbols and the other with a composition dependent sublimation energy according to eq. 10 depicted as filled symbols in Fig. 13. In both cases sublimation leads to material loss from the surface but the strong Be influx $\left(\approx 5.0 \times 10^{18}\left(\mathrm{~m}^{-2} \mathrm{~s}^{-1}\right)\right)$ at this location compensates the sublimation loss. The Be diffusion leads to the formation of an $\approx 50 \mathrm{~nm} \mathrm{Be}{ }_{12} \mathrm{~W}$ mixed layer at a depth of $\approx 150 \mathrm{~nm}$ in Fig. 13. One can therefore conclude that at the moderate steady state temperatures diffusion is low on Be is continuously deposited resulting in a thick layer. But during a temperature excursion diffusion in combination with the high Be influx from the plasma leads to the transport of Be into the $\mathrm{W}$ bulk where Be/W alloys can be formed. 
The reason that there is so little difference between the two sublimation models is that despite the fact that sublimation with a constant sublimation energy of $3.2 \mathrm{eV}$ is much higher than for the composition dependant sublimation model, the sublimation loss from the surface for the maximum temperature considered here is rather small. The case with constant sublimation energy results in a negligible lower Be concentration in the SZ due to the higher sublimation rate in this case.

It can thus be concluded that independent of the sublimation model used, the diffusion during temperature excursions can locally lead to the formation Be rich Be/W alloys. Of course this depends on the detailed balance of deposition, diffusion and sublimation and will not occur everywhere. At much higher temperatures any deposited Be will melt.

\section{Conclusions}

The Be flux distribution in a full W ITER divertor due to Be erosion at the main wall has been calculated for a high power and density ITER discharge with Ar as main radiating impurity. The calculations included the effects of the re-erosion and re-deposition of Be. The highest Be flux fractions are found at the inner divertor entrance baffle and at the dome baffle. Thick Be layers can only grow close to the inner divertor entrance baffle and at the dome baffle where the incident Be fluxes can compensate the re-erosion of Be. Under steady state conditions the surface temperatures at these areas is too low as to allow for significant diffusion and thus no formation of $\mathrm{Be} / \mathrm{W}$ alloys is expected. However based on the currently available thermodynamic data for the Be/W system the calculations show that during temperature excursions the diffusion can be strong enough to lead to the formation of Be rich Be/W alloys in some locations (e.g. the inner divertor leg). The sublimation flux of Be off the surface is not strong enough to inhibit the diffusion of Be into the $\mathrm{W}$ bulk for temperatures below the Be melting temperature. 
[1] M. Mayer, R. Behrisch, H. Plank, J. Roth, G. Dollinger, and C. M. Frey. J. Nucl. Mater., 230:67, 1996.

[2] M. Mayer. J. Nucl. Mater., 240:764, 1997.

[3] R. A. Causey and D. S. Walsh. J. Nucl. Mater., 254:84, 1998.

[4] A. V. Markin, V. P. Dubkov, A. E. Gorodetsky, and M. A. Negodaev. J. Nucl. Mater., 283:1094, 2000.

[5] M.J. Baldwin, K. Schmid, R.P. Doerner, A. Wiltner, R. Seraydarian, and Ch. Linsmeier. J. Nucl. Mater., 337:590, 2005.

[6] Ch. Linsmeier, K. Ertl, J. Roth, A. Wiltner, K. Schmid, F. Kost, S.R. Bhattacharyya, M. Baldwin, and R.P. Doerner. J. Nucl. Mater., 363-365, 2007.

[7] R.P. Doerner, M.J. Baldwin, and R.A. Causey. J. Nucl. Mater., 342:63, 2005.

[8] J. N. Brooks, J. P. Allaina, D. A. Almanb, and D. N. Ruzicb. Fusion Engineering and Design, 72:363, 2005.

[9] A. Kirschner, D. Borodin, S. Droste, V. Philipps, U. Samm, G. Federici, A. Kukushkin, and A. Loarte. J. Nucl. Mater., 363-365, 2007.

[10] P.C. Stangeby and J. D. Elder. J. Nucl. Mater., 196:258, 1992.

[11] A.S.Kukushkin and H.D.Pacher. Plasma Phys. Control. Fusion, 44:931, 2002.

[12] K. Schmid and J. Roth. J. Nucl. Mater., 313:302, 2003.

[13] W. Möller, W. Eckstein, and J. P. Biersack. Computer Physics Communications, 51 No. 8:355, 1988.

[14] C.B. Alcock, V.P. Iktin, and M.K. Horrigan. Can. Metall. Quat., 23:309, 1984.

[15] J. Bohdansky. Nucl. Inst. Meth. B, 2:587, 1984.

[16] W. Eckstein, M. Hou, and V.I. Shulga. Nucl. Instrum. Methods B, 477-486:119, 1996.

[17] H. Ibach and H Lüth. Festkörperphysik. Springer Verlag, 1999.

[18] K. Schmid and J. Roth. J. Nucl. Mater., 302:96, 2002.

[19] M.J. Baldwin, R.P. Doerner, D. Nishijima, D. Buchenauer b, W.M. Clift, R.A. Causey, and K. Schmid. J. Nucl. Mater., 363-365, 2007.

[20] R. Behrisch, G. Federici, A. Kukushkin, and D. Reiter. J. Nucl. Mater., 313:388, 2003.

[21] R.D. Monk and JET Team. Nuclear Fusion, 39:1751, 1999.

[22] V. Barabash, G. Federici, J. Linke, and C.H. Wu. J. Nucl. Mater., 313-316:42, 2003.

[23] T.B. Massalski, H. Okamoto, P.R. Subramanian, and L. Kacprzak. Binary Alloy Phase Diagrams, 2nd Ed. ASM International, 1996. 


\section{Figure captions}

Fig. 1

Subdivision of the ITER divertor for the equilibrium flux calculations. The element numbering derives from the internal DIVIMP wall element numbering scheme.

Fig. 4

DIVIMP calculation grid and first wall geometry. Also shown is the origin for wall indexing and the poloidal distance used in some of the other plots.

Fig. 5

Maximum distance between DIVIMP calculation grid and the first wall.

Fig. 2

Non linear dependence of the Be sputter yield from a Be/W mixed surface on the Be concentration in the sputter and reaction zone.

Fig. 3

Comparison of Be depth profiles as calculated by TRIDYN and ERODEPDIF. The conditions were choosen such that sublimation and diffusion are negligible so that the calculation results were comparable.

Fig. 6

The flux redistribution matrix $\xi_{i j}$ integrated over all charge states for simplicity.

Fig. 7

D ion energies (a.) and ion flues (b.) at the first wall extrapolated from calculation grid boundary of the B2/Eirene background plasma.

Fig. 8

Average Ar ion energies (a.) and ion flues (b.) at the first wall extrapolated from calculation grid boundary of the B2/Eirene background plasma.

Fig. 9

Average D-CX energies (a.) and D-CX flues (b.) at the first wall extrapolated from calculation grid boundary of the B2/Eirene background plasma.

Fig. 10

Be erosion fluxes at the ITER main wall due to D, D-CX, $\mathrm{He}^{+x}$ and $\mathrm{Ar}^{+x}$ bombardment. Fig. 11

Be flux fraction in the ITER divertor. Square symbols show the charge resolved Be flux distribution due to main wall erosion alone. Filled circles show the charge integrated Be flux distribution including the effect of re-erosion and re-deposition.

Fig. 12

Deposition (square symbols) and erosion (cirlces) rates in the ITER divertor: Empty symbols: due to main wall erosion alone, filled symbols: including the influence of reerosion and re-deposition.

Fig. 13

Be depth profiles in $\mathrm{W}$ before and after a temperature excursion. The profiles after the excursion we calculated with two different sublimation models. 


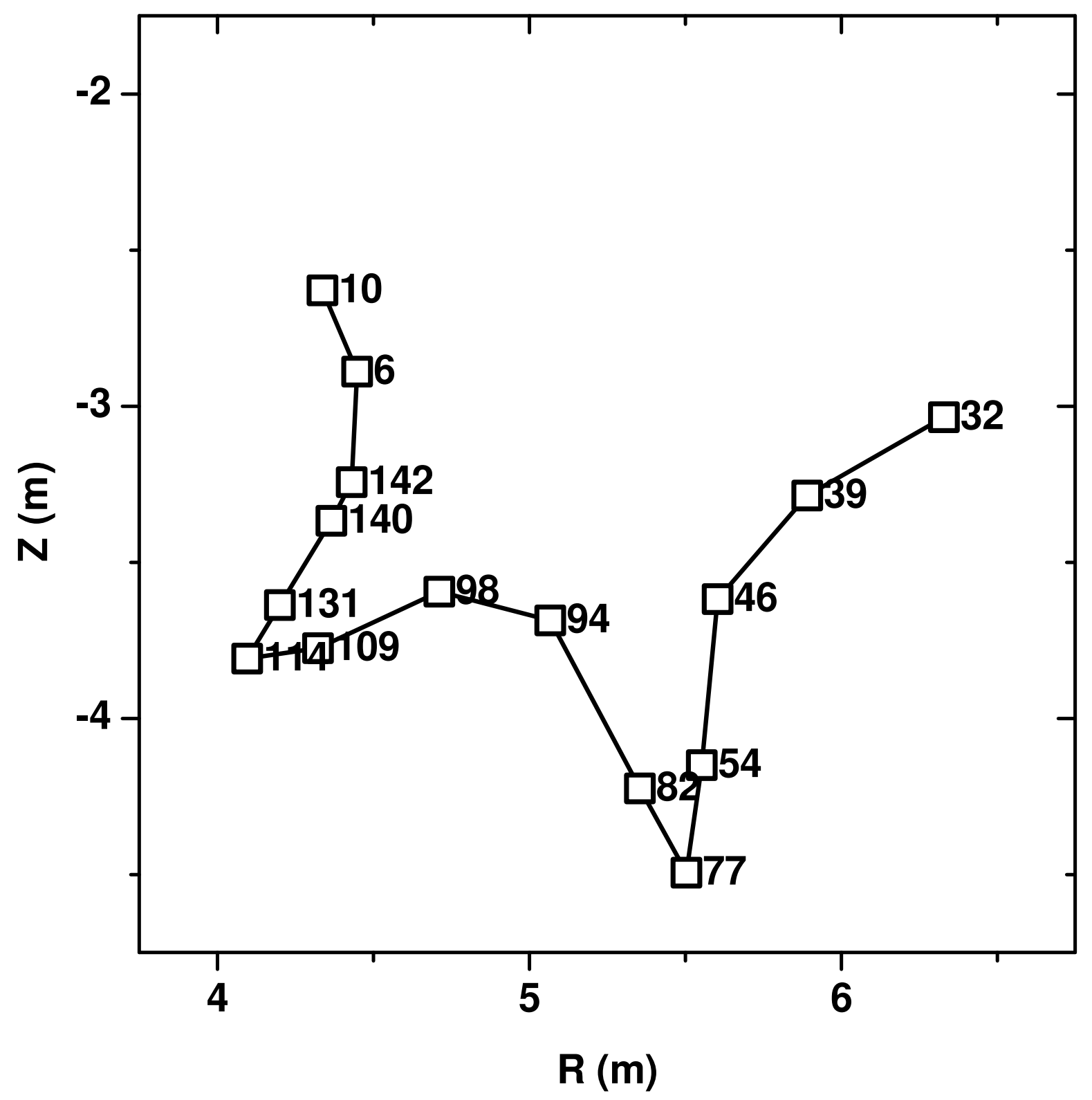

Figure 1. 


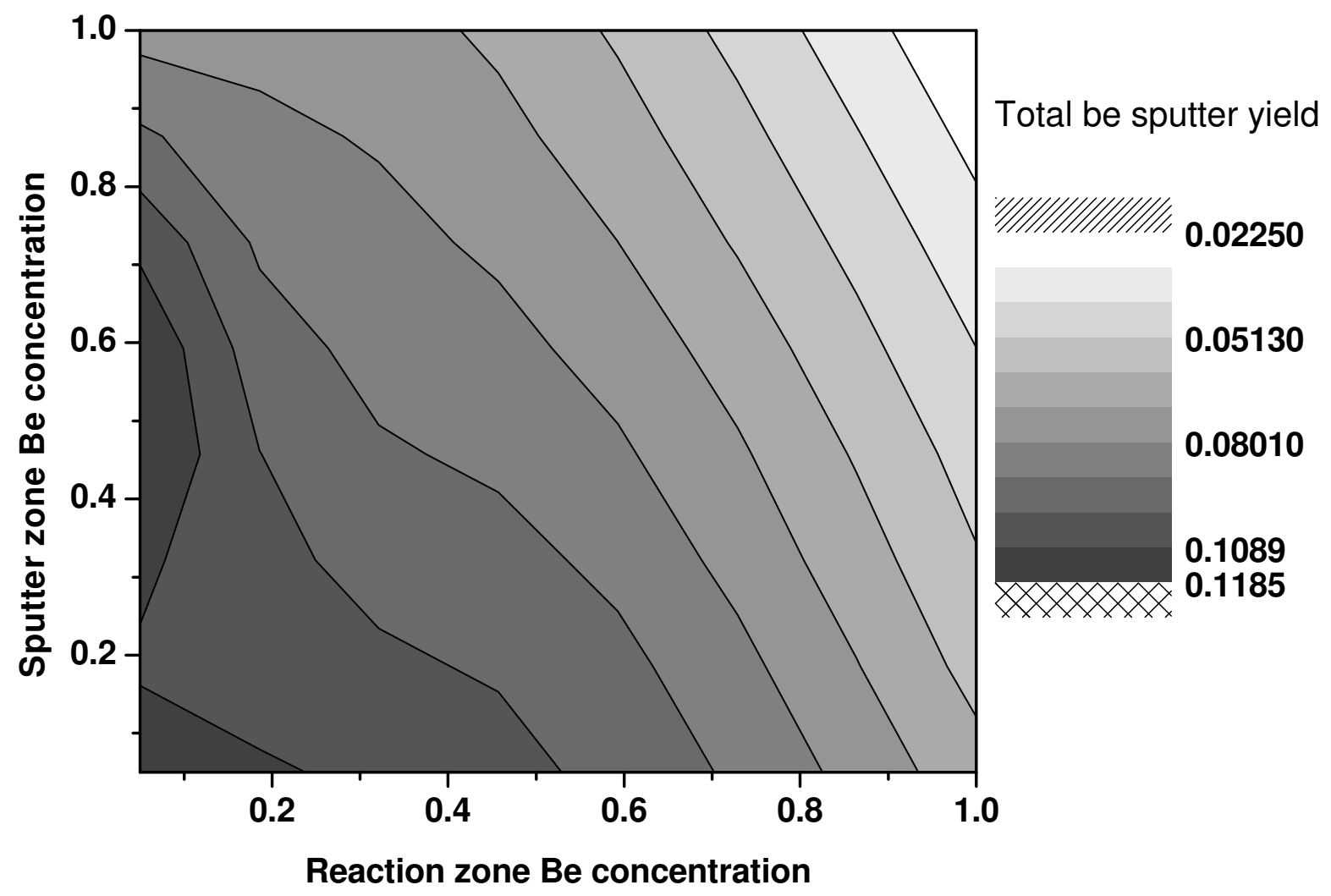

Figure 2.

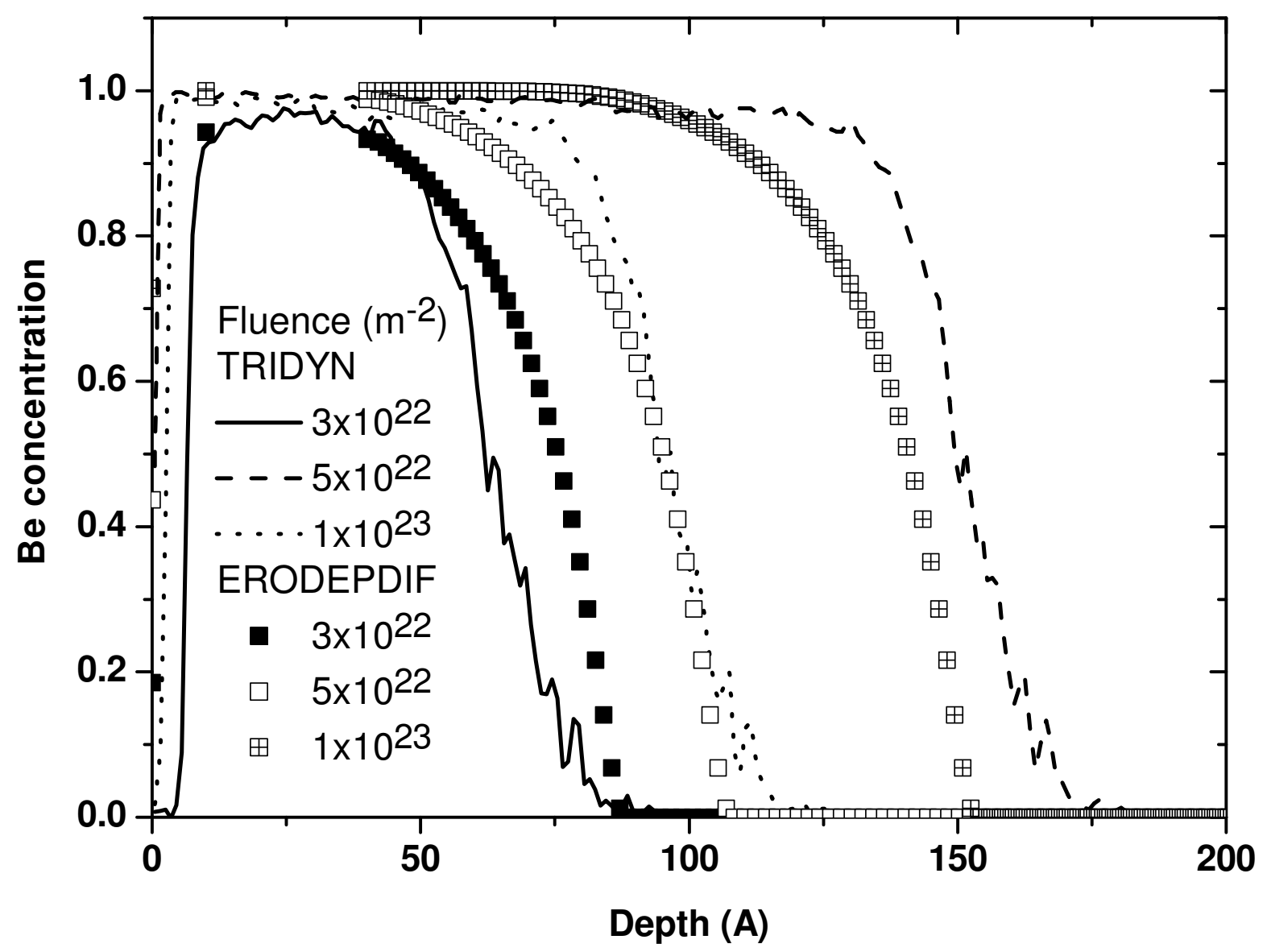

Figure 3. 


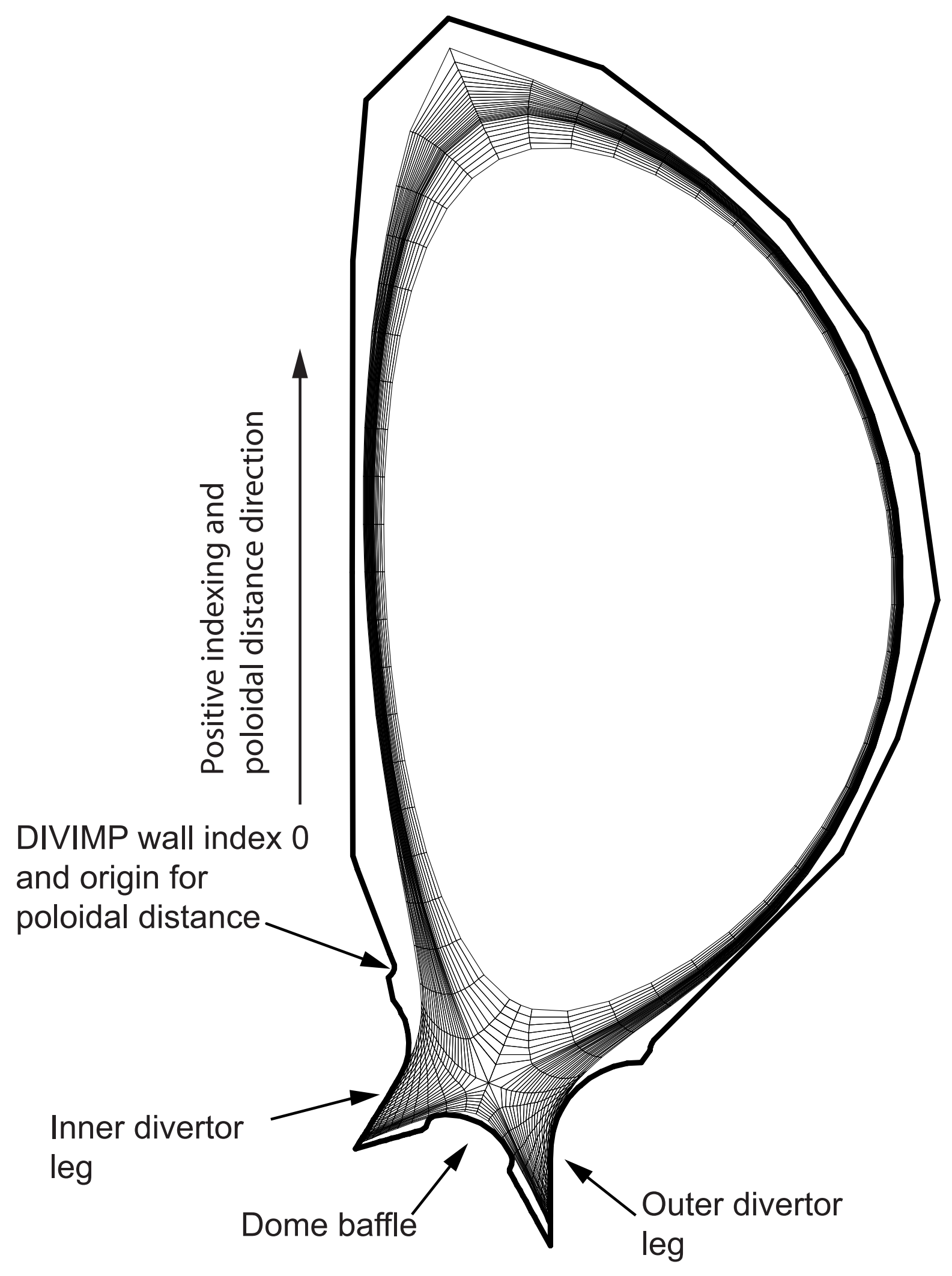

Figure 4 . 


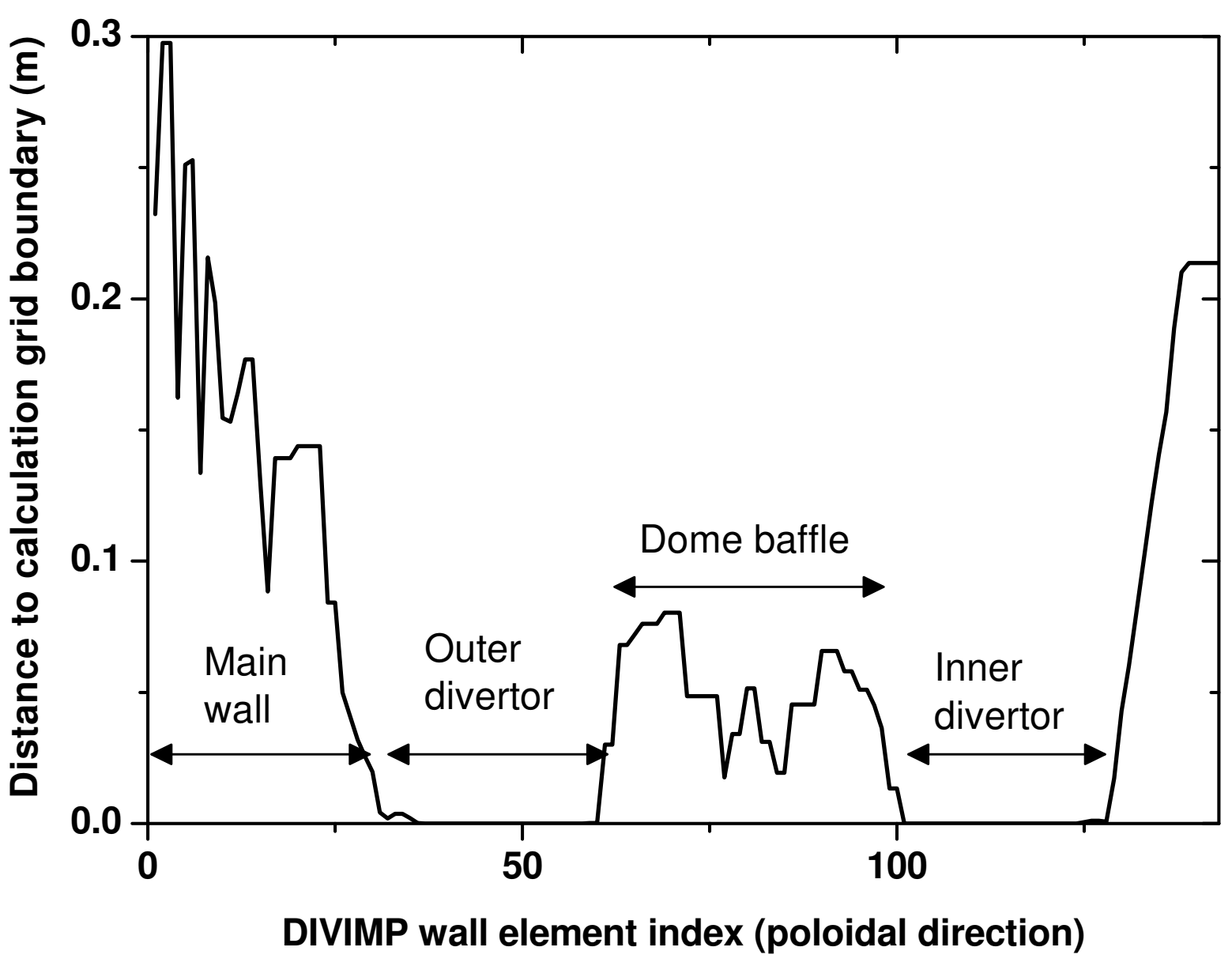

Figure 5.
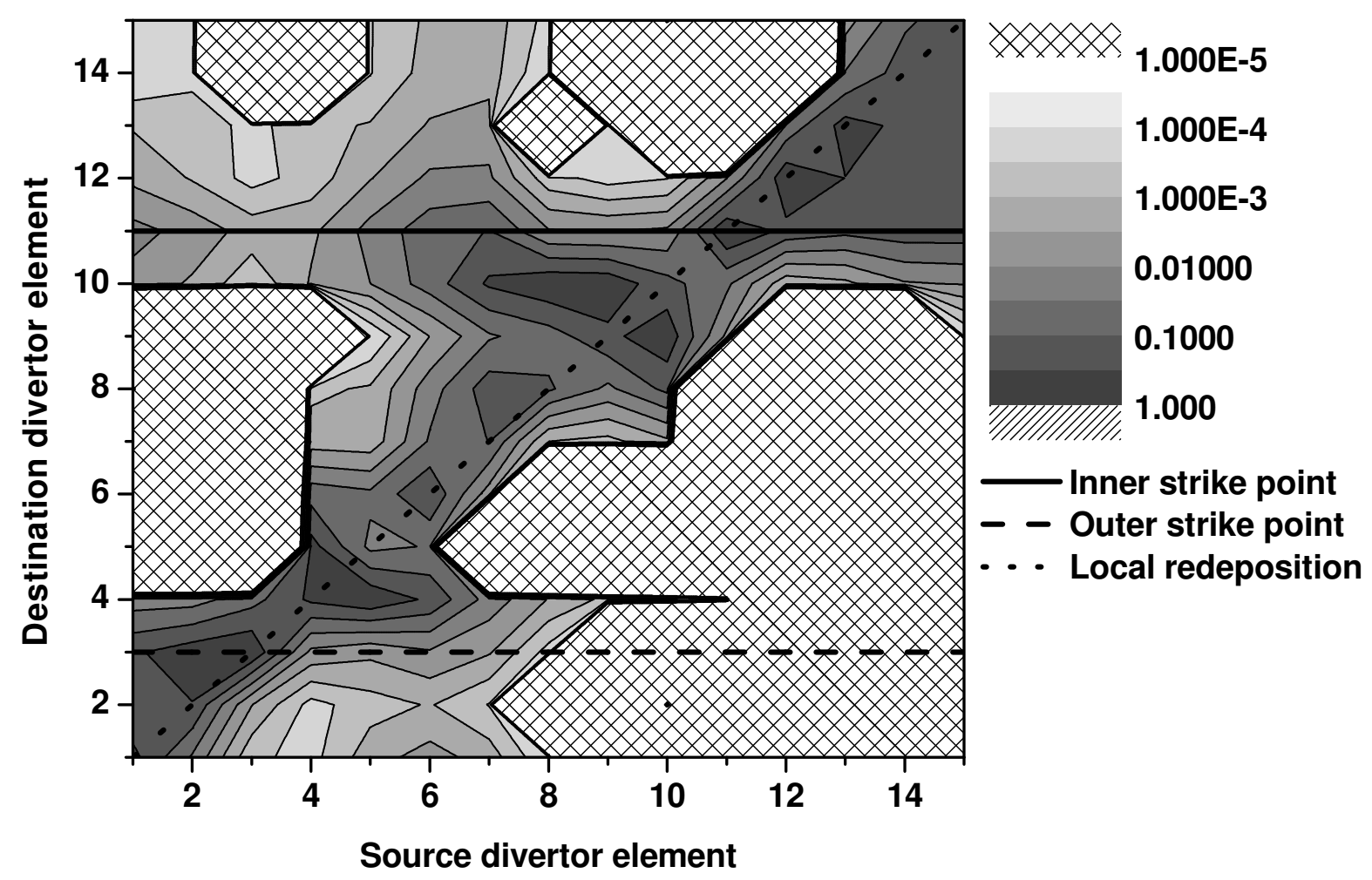

Figure 6. 

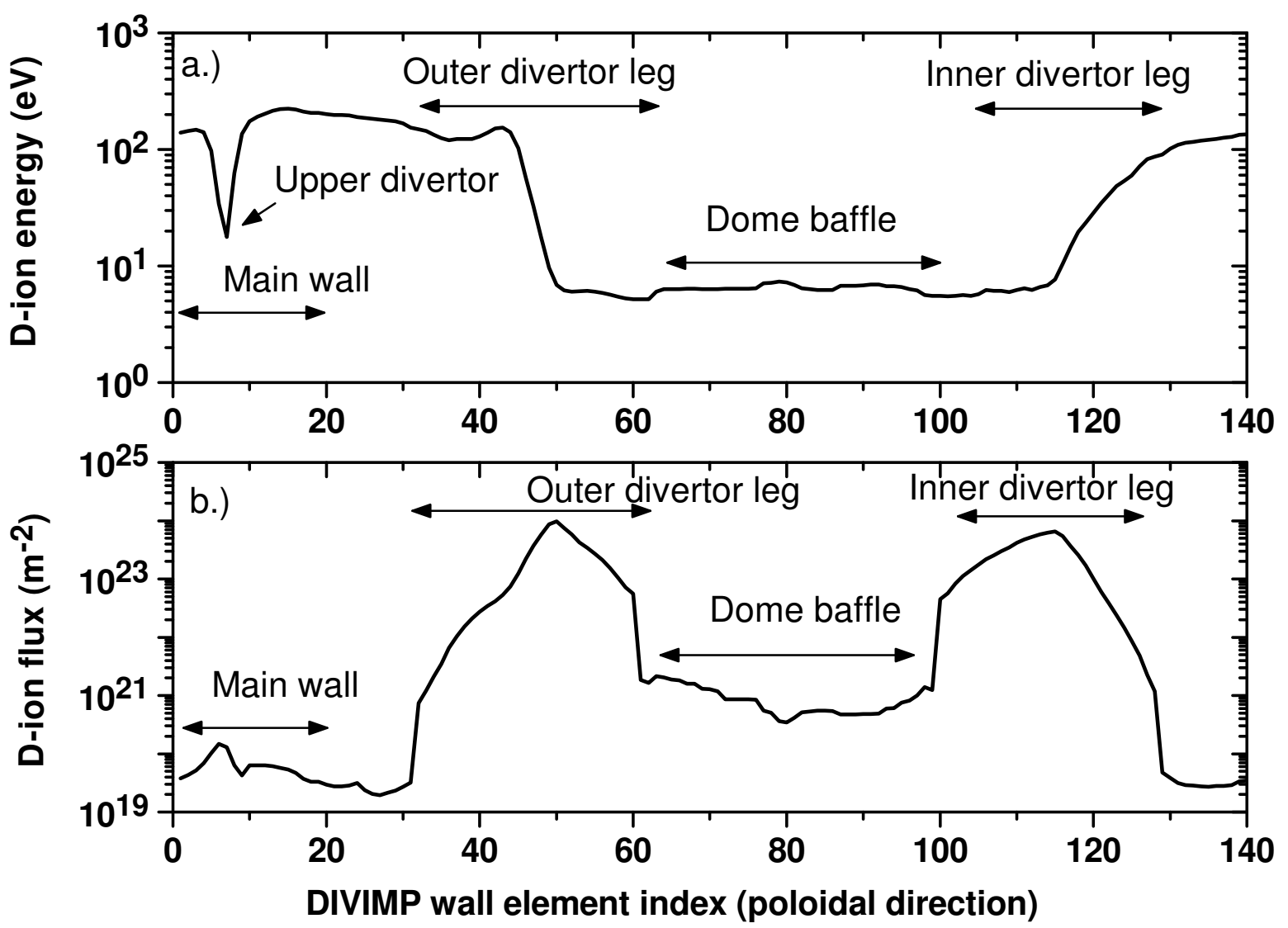

Figure 7 . 

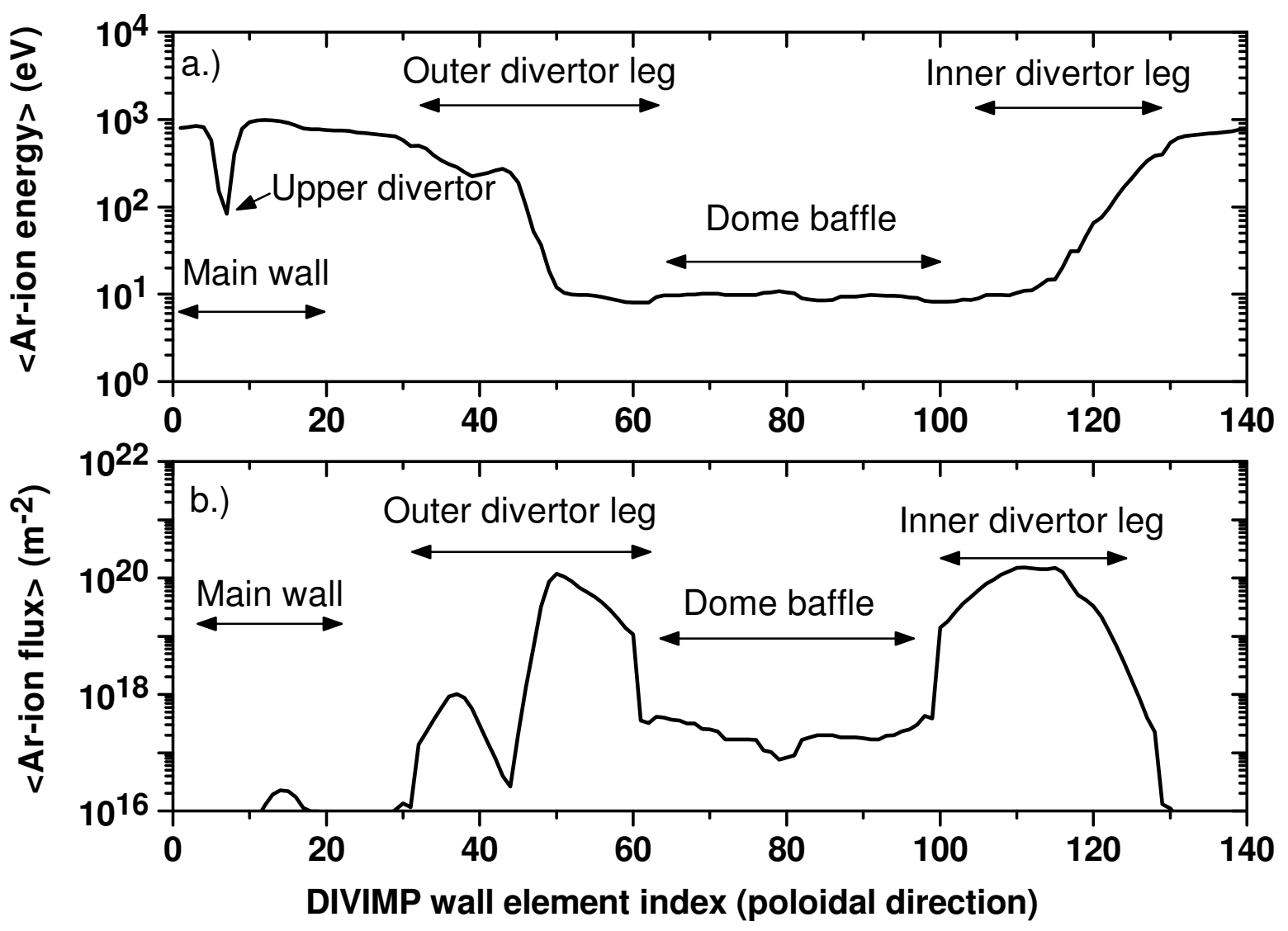

Figure 8. 

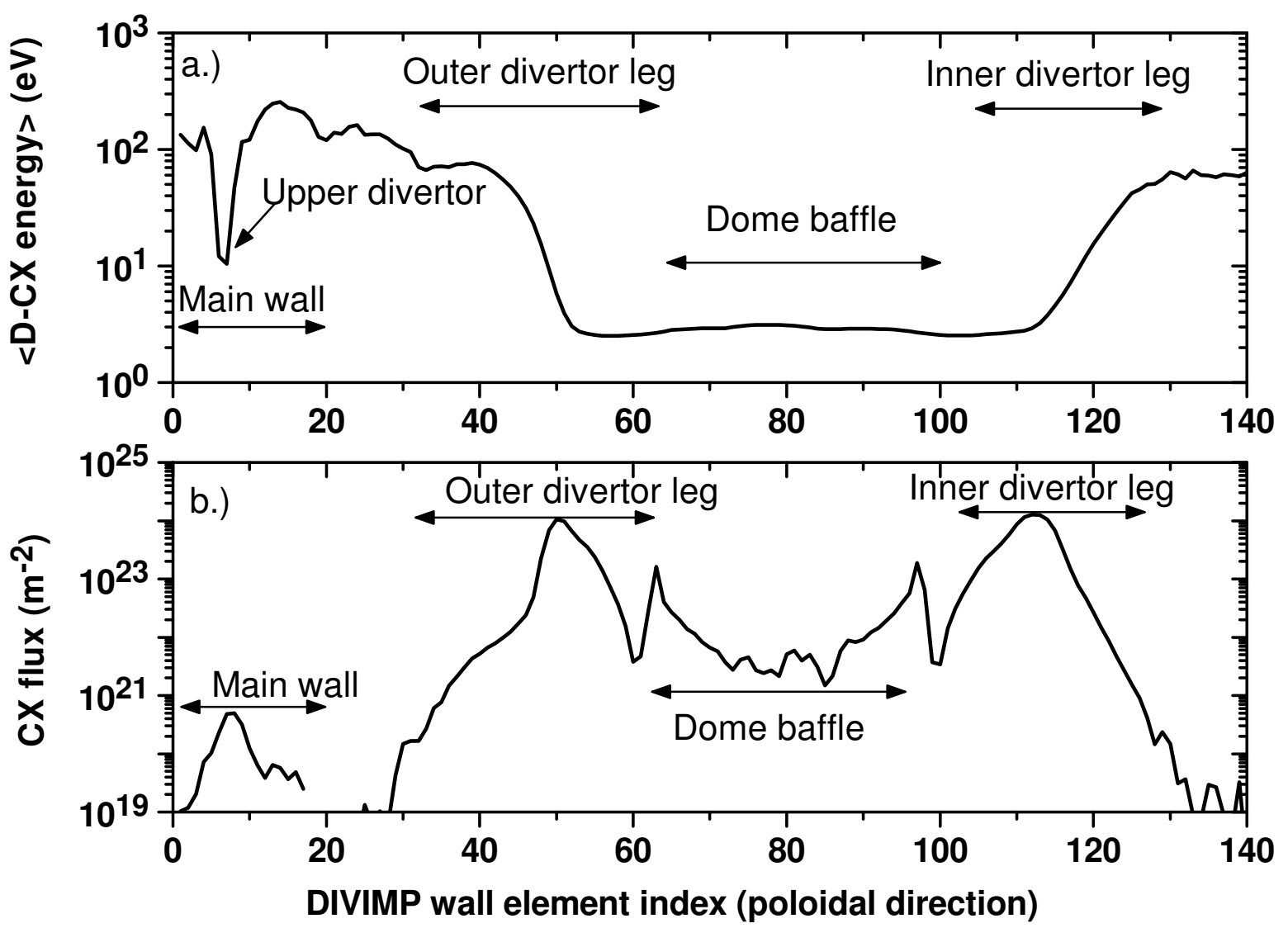

Figure 9.

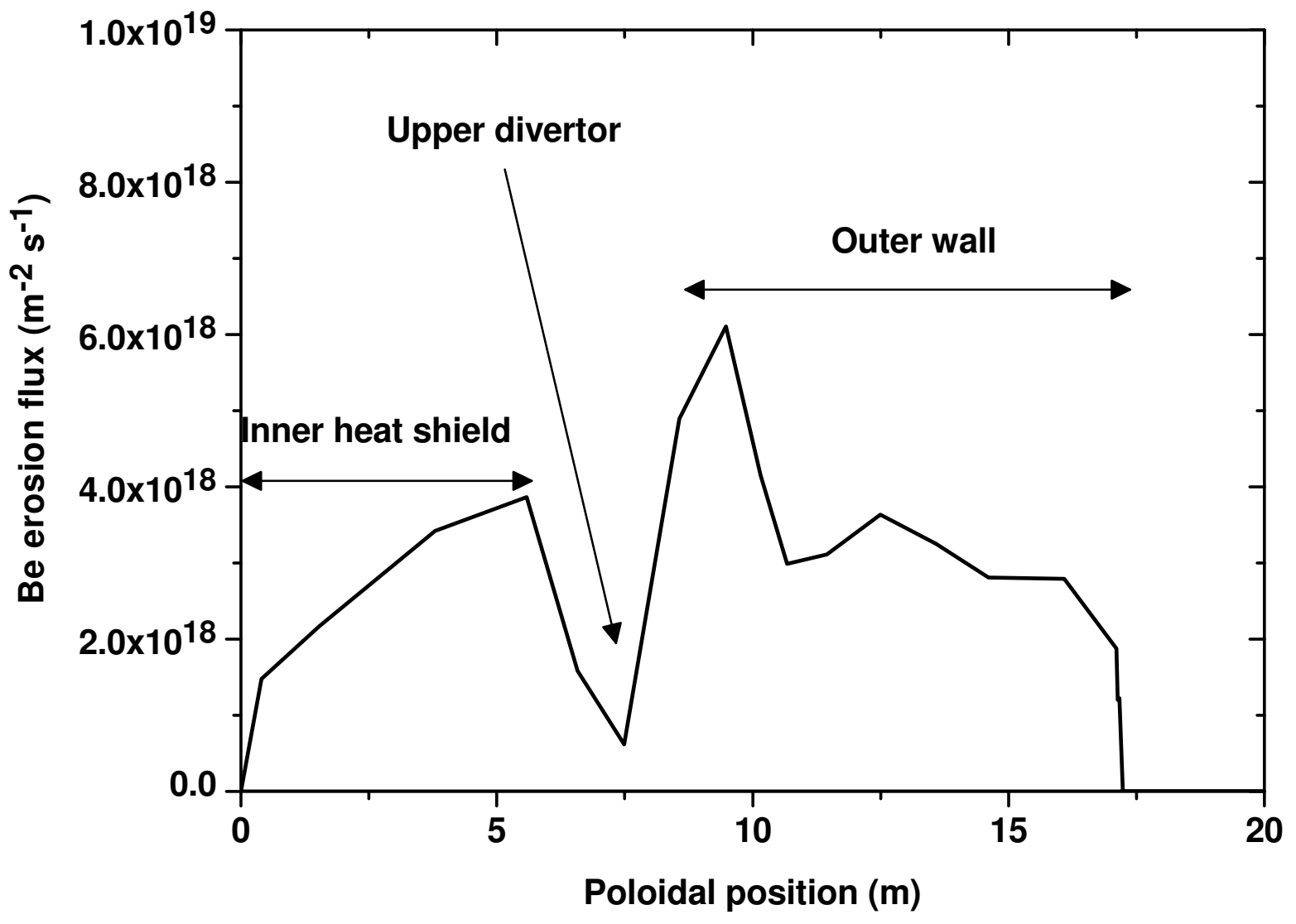

Figure 10. 


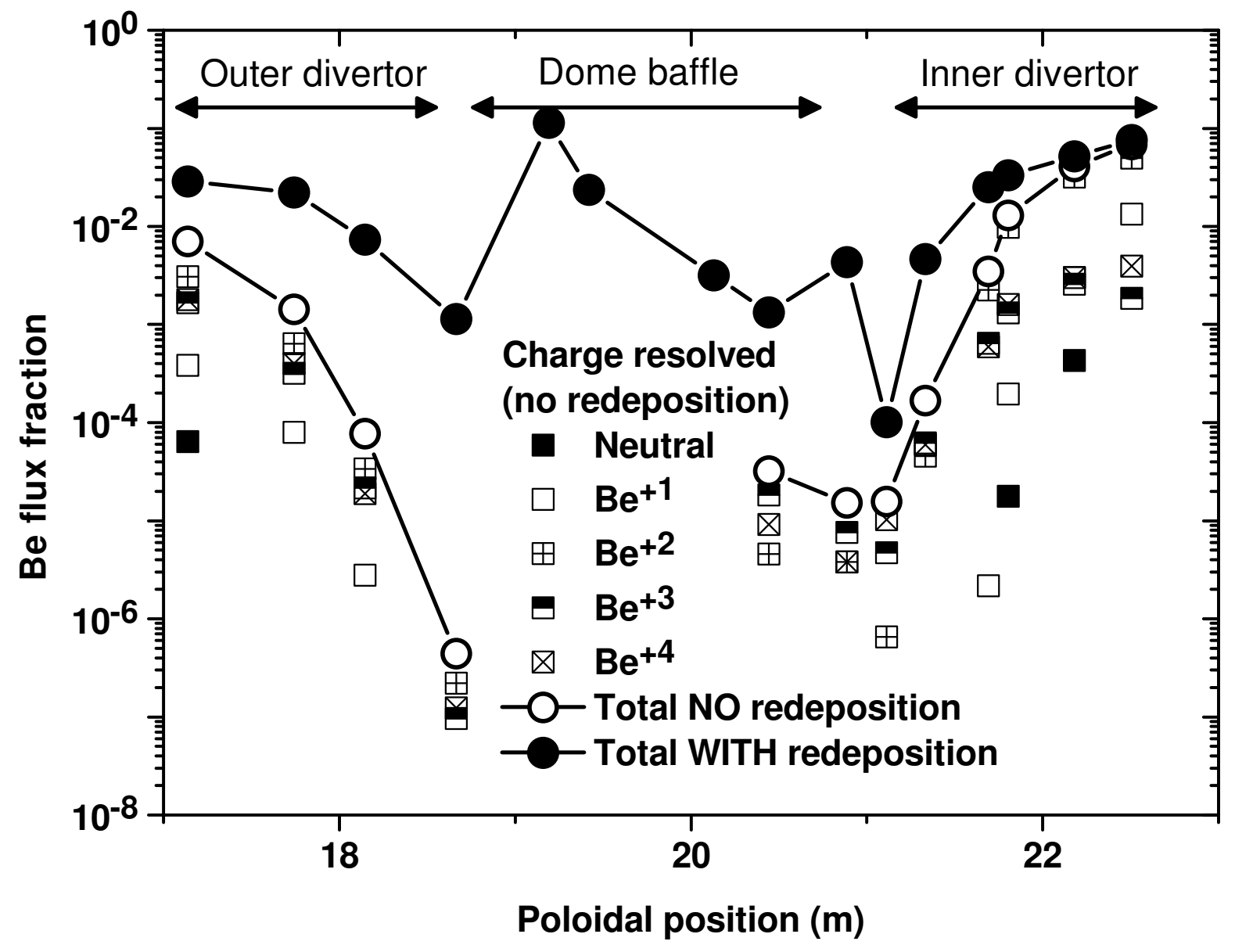

Figure 11. 


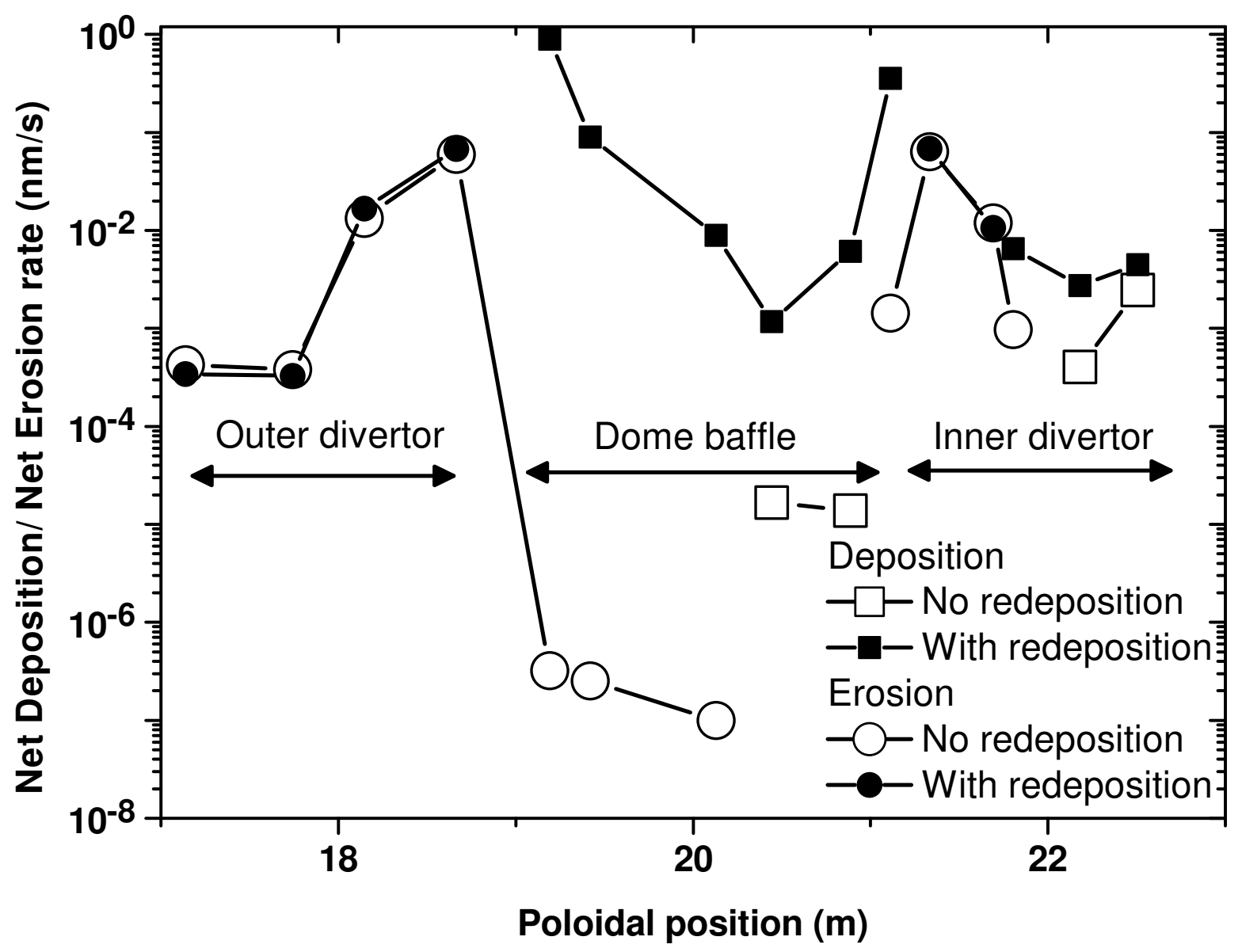

Figure 12. 


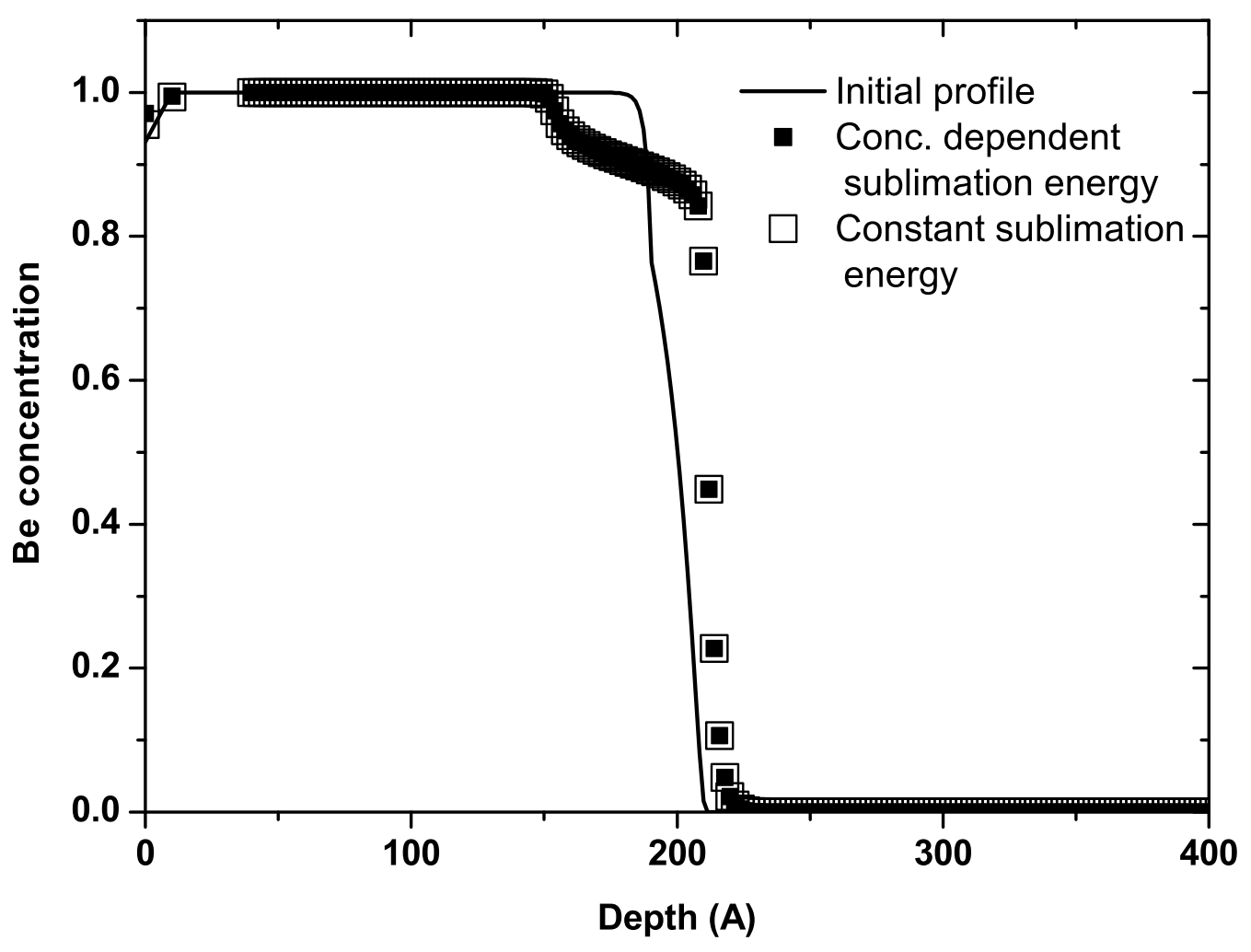

Figure 13. 